\title{
Evaluación de la Pérdida Osea y del Éxito / Fracaso de Implantes de Titanio de Posicionamiento Inmediato y Tar- dío con Carga Inmediata, Usados para Restauraciones de Arco Completo a 12 Meses de su Implantación
}

\author{
Evaluation of Bone Loss and Success/Failure of Titanium Implant Positioning with \\ Immediate and Late Charge, Used in Full Arch Restorations at 12 Months of Implant
}

María Dolores Guerrero*; Gonzalo Cid ${ }^{*}$ \& Patricio Oliva*

GUERRERO, M. D.; CID, G.; OLIVA, P. Evaluación de la pérdida ósea y del éxito / fracaso de implantes de titanio de posicionamiento inmediato y tardío con carga inmediata, usados para restauraciones de arco completo a 12 meses de su implantación. Int. J. Odontostomat., 10(3):399-407, 2016.

RESUMEN: El propósito de este estudio fue evaluar el nivel óseo y el éxito y fracaso de implantes con carga inmediata posicionados inmediatamente después de la extracción para rehabilitaciones de arco completo y compararlos con los resultados obtenidos con implantes situados en alvéolos cicatrizados, en un periodo de 12 meses, para determinar la mejor alternativa de uso. Se efectúo una revisión sistemática en base a ensayos clínicos aleatorios prospectivos y los ensayos clínicos aleatorizado o no aleatorizados, controlados o no controlados que evaluaron el fracaso y reabsorción ósea de implantes con carga inmediata posicionados en alveolos post-extracción y en alvéolos cicatrizados en pacientes adultos para rehabilitaciones de arco completo. Los datos de los ensayos clínicos fueron ingresados al software Review Manager®. Se realizaron dos metaanálisis para las distintas variables a analizar y posteriormente un análisis de sensibilidad para eliminar los estudios que causaban sesgo. De un total de 431 implantes analizados, 16 de ellos fracasaron, 11 de los cuales habían sido posicionados inmediatamente luego de la exodoncia. Tres de los autores considerados en la revisión, concuerdan en que hay un mayor éxito y menor pérdida ósea en aquellos implantes posicionados de manera tardía. Para rehabilitaciones implanto-soportadas de arco completo fijas o removibles, el protocolo de posicionamiento tardío, demostró ser una mejor alternativa puesto que manifestó una menor tasa de fracaso y una menor pérdida ósea.

PALABRAS CLAVE: restauración de arco completo, implante dental, reabsorción alveolar, carga inmediata, posicionamiento inmediato.

\section{INTRODUCCIÓN}

Los implantes son una opción terapéutica frente a la pérdida o ausencia de dientes (Grunder, 2001), se define como el anclaje de material aloplástico en los maxilares para crear elementos de soporte y sujeción, con el fin de sustituir dientes perdidos (Spiekermann et al., 2001).

El cuerpo del implante debe anclarse al tejido óseo mediante cicatrización con anquilosis y sin tejido conectivo, es decir; osteointegrarse (Spiekermann et al.). El éxito de esta conexión o interfase huesoimplante depende de factores biológicos y sistémicos del paciente, de las características del implante y su superficie (Venegas-Acosta et al., 2010). Los factores que interfieren en la osteointegración, responden a cuatro conceptos claves: Ajuste correcto del implante en el lecho óseo, técnica quirúrgica cuidadosa, diseño del implante y calidad del tejido óseo (Sáenz et al., 2006).

Los implantes dentarios podrán ser clasificados como "inmediatos" cuando se colocan en el alvéolo inmediatamente tras la extracción del diente, "inmediatos-tardíos" cuando se colocan después de

*Facultad de Ciencias de la Salud, Universidad del Desarrollo, Concepción, Chile. 
GUERRERO, M. D.; CID, G.; OLIVA, P. Evaluación de la pérdida ósea y del éxito / fracaso de implantes de titanio de posicionamiento inmediato y tardío con carga inmediata, usados para restauraciones de arco completo a 12 meses de su implantación. Int. J. Odontostomat., 10(3):399-407, 2016.

semanas o meses para permitir la cicatrización de los tejidos blandos y como implantes "tardíos" que son aquellos que se posicionan en el hueso una vez que éste está parcial o totalmente cicatrizado (Esposito et al., 2010). Los implantes inmediatos suelen indicarse para la sustitución de dientes con patologías sin posibilidad de tratamiento. Entre sus ventajas, respecto a los implantes diferidos, están el disminuir la reabsorción ósea del alvéolo post-extracción, acortar el tiempo de tratamiento rehabilitador, evitar una segunda cirugía de implantación (Peñarrocha et al., 2004) y permitir conservar la estructura de la papila gingival (Grunder). Sus inconvenientes principales en comparación con los inmediatos-tardíos y tardíos, radican en la necesidad de efectuar, técnicas de regeneración ósea guiada con membranas, con el riesgo de exposición e infección de la misma; y la necesidad de injertos mucogingivales para el cierre del alvéolo y/o cubrir las membranas

Otra forma de clasificar los implantes es catalogarlos como abiertos y cerrados, siendo los primeros aquellos en que el implante posicionado en el hueso tiene contacto directo con la cavidad oral a través de un muñón protésico. Por su parte, los implantes cerrados no toman contacto directo con la cavidad oral. A su vez, existen dos sistemas de implantación, bifásicos y monofásicos (Spiekermann et al.). El sistema bifásico requiere de dos tiempos quirúrgicos, habiendo una primera fase en la que se anclan los implantes al hueso, permitiendo mantener una etapa de cicatrización libre de cargas y sin ningún tipo de alteración previo a la segunda etapa, en la que se ejecuta la apertura quirúrgica del implante al medio oral para efectuar el tratamiento protésico, por otra parte (Spiekermann et al.), los sistemas monofásicos tienen un solo tiempo quirúrgico, en el que los implantes son anclados al hueso y sus pilares protésicos están expuestos a la cavidad oral inmediatamente después de la cirugía, es por esto que éste sistema requiere de una estabilización inmediata para evitar el exceso de cargas durante el periodo de reparación del hueso (Spiekermann et al.). El implante puede recibir, carga inmediata: cuando se establece función oclusal durante la primera semana tras el posicionamiento del implante; carga temprana: cuando esto se realiza entre una semana y dos meses después del posicionamiento del implante o puede ser cargado convencionalmente cuando las fuerzas se reciben dos meses después de la implantación (Esposito et al., 2007).
El éxito de un tratamiento de implantes varía entre el 90 y el $99 \%$, dependiendo del tiempo de observación de éstos (Jiménez-López, 1998). Para determinar el éxito clínico no debe pesquisarse dolor, signos de infección ni movilidad (debe tener estabilidad individual) (Wolfinger et al., 2003) (Barbier et al., 2012) (Deng et al., 2010). Mientras que para definir el éxito radiográfico, debe observarse osteointegración, es decir, ausencia de un halo radiolúcido peri-implantario. (Wolfinger et al.; Barbier et al.; Deng et al.; Peñarrocha et al.). Actualmente se considera que un implante fue correctamente posicionado cuando experimenta una pérdida ósea horizontal de aproximadamente $1,5 \mathrm{~mm}$ durante el primer año desde su inserción. Éste fenómeno se continúa con el pasar de los años, produciéndose reabsorciones que pueden ir de los $0,1 \mathrm{a} 0,2 \mathrm{~mm}$ al año (Spiekermann et al.).

Frente al deterioro de la dentición residual como consecuencia de caries y / o un soporte periodontal reducido, es necesario evaluar las mejores opciones de tratamiento. Analizar si es más favorable conservar y utilizar los dientes remanentes, una sobredentadura implanto-soportada (Jiménez-López) o una reconstrucción implanto soportada fija. En éste tipo de tratamientos la aplicación de carga inmediata en implantes posicionados inmediatamente después de la exodoncia sugiere una gran ventaja, ya que en caso contrario, para evitar cargar los implantes y/o permitir la cicatrización de los alveolos, el paciente no debe llevar prótesis removible en absoluto durante 2 semanas tras la cirugía y luego de esto debe utilizar una prótesis total como provisional durante varios meses (Grunder). Sumado a lo anterior, si se ha planificado una reconstrucción implanto-soportada fija, el uso de la prótesis total provisoria traerá como consecuencia la pérdida total de las papilas (Grunder), afectando de manera importante la estética.

El objetivo de esta revisión de la literatura fue evaluar la pérdida ósea y la supervivencia de implantes con carga inmediata, posicionados de manera inmediata en contraste con aquellos cargados inmediatamente pero posicionados de manera tardía, cuando son utilizados para realizar restauraciones de arco completo, buscando responder a la pregunta de investigación que motivó éste estudio: ¿El posicionamiento de implantes cargados inmediatamente, usados en restauraciones de arco completo, en alveolos frescos o cicatrizados tiene incidencia en la pérdida ósea experimentada y a la vez en el éxito o fracaso del tratamiento en sí? 
GUERRERO, M. D.; CID, G.; OLIVA, P. Evaluación de la pérdida ósea y del éxito / fracaso de implantes de titanio de posicionamiento inmediato y tardío con carga inmediata, usados para restauraciones de arco completo a 12 meses de su implantación. Int. J. Odontostomat., 10(3):399-407, 2016.

\section{MATERIAL Y MÉTODO}

Se efectúo una revisión sistemática con metaanálisis centrados en ensayos clínicos. Sin restricción de idioma y publicados entre el año 1997 y Junio de 2014.

Tipos de estudios. Se eligieron ensayos clínicos aleatorios prospectivos y los ensayos clínicos aleatorizados o no aleatorizados, controlados o no controlados, que estén publicados y en curso y evaluaran tras 12 meses el éxito, fracaso y reabsorción ósea de implantes con carga inmediata posicionados en alveolos post-extracción y en alvéolos cicatrizados en pacientes adultos para rehabilitaciones de arco completo.

Tipo de participantes. Se incluyeron los ensayos clínicos cuya muestra estuviese basada en pacientes con necesidad de restauraciones de arco completo implanto-soportadas. Los implantes debían recibir carga inmediata y a la vez deben posicionarse tanto en alvéolos frescos como cicatrizados.

Tipo de intervención. Restauraciones de arco completo implanto-soportadas con carga inmediata y de posicionamiento inmediato.

\section{Tipos de Medidas de Resultados}

Intervención: Implantes de titanio usado para soportar restauraciones de arco completo, cargados inmediatamente y posicionados en alvéolos post-extracción o en alvéolos cicatrizados.

\section{Criterios para establecer éxito del implante:}

-Ausencia clínica de movilidad, estabilidad individual. -Ausencia de dolor o signos de infección.

-Ausencia de peri-implantitis o radiolucidez circundante al implante.

\section{Controles radiográficos:}

-Radiografías con técnica del cono largo (paralelismo). -Reabsorción ósea medida desde baseline.

Estrategia de Búsqueda para la Identificación de Estudios: Se realizaron búsquedas en el registro de MEDLINE vía PubMed (desde el año 2000 hasta mayo del año 2014). Adicionalmente se efectuó una búsqueda en EBSCO de las publicaciones no disponibles en
MEDLINE, complementándose a la vez con una búsqueda de ensayos clínicos en el Registro Cochrane Central de Ensayos Controlados (CENTRAL), en Clinicaltrial.gov (ambas buscando publicaciones hasta mayo del 2014). También se buscaron estudios específicos en el sitio WEB OF SCIENCE (desde 1997 a junio de 2014)

Se estableció contacto con los investigadores y grupos de investigación pertinentes locales e internacionales (Grupo de Salud Oral de la Cochrane) para determinar la posibilidad de acceder a ensayos clínicos publicados o no publicados.

La búsqueda se concentró en las siguientes opciones de búsqueda:

a) Registro de ensayos de CENTRAL MEDLINE PUBMED, EBSCO, Registro Cochrane Central de Ensayos Controlados y WEB OF SCIENCE.

b) Búsqueda en bases de datos electrónicas.

c) Registro de ensayos del grupo de salud oral de la Cochrane.

Todas las referencias señaladas por la estrategia de búsqueda fueron revisadas por dos autores, que de forma independiente revisaron y juzgaron si los ensayos clínicos cumplían con los criterios de inclusión. Los desacuerdos se resolvieron por discusión y en casos de desacuerdo se solicitó a un tercer autor dirimir las diferencias.

Análisis estadístico: La información obtenida desde los artículos originales fue ingresada en el software Review Manager® versión 5.2 (RevMan). Se realizaron dos metaanálisis para estudiar las variables de éxito/ fracaso y pérdida ósea entre los grupos caso y control. En ambos casos se utilizó modelos de efecto fijo, ya que soporta estudios con muestras pequeñas y escasos eventos considerando que la variabilidad de los estudios es producida por el azar. Los resultados se ajustaron mediante la aplicación de la prueba Mantel Haenszel, para el análisis de las variables dicotómicas (éxito/fracaso), y de Varianza Inversa en el caso de las variables continuas (pérdida ósea). Para ambos análisis se utilizó un intervalo de confianza del 95\%.

El análisis de heterogeneidad se evaluó mediante el test de 12, considerando los valores de 25,50 y $75 \%$ como baja, moderada y alta heterogeneidad. Pese a la alta heterogeneidad observada en los análi- 
GUERRERO, M. D.; CID, G.; OLIVA, P. Evaluación de la pérdida ósea y del éxito / fracaso de implantes de titanio de posicionamiento inmediato y tardío con carga inmediata, usados para restauraciones de arco completo a 12 meses de su implantación. Int. J. Odontostomat., 10(3):399-407, 2016.

sis, sólo fue posible realizar el análisis de sensibilidad evaluación del éxito y fracaso de los implantes, pues para el análisis de nivel óseo sólo se dispuso de 3 estudios que cumplían con los criterios de inclusión. El análisis de sensibilidad fue efectuado para determinar el estudio que producía la heterogeneidad.

\section{RESULTADOS}

De los 11 estudios seleccionados en un principio, sólo 4 cumplían con los criterios de inclusión. Los estudios incluyeron la información del número total de implantes posicionados y cuántos de estos habían fracasado según los criterios estipulados por los autores al controlarlos a los 12 meses después de su implantación en el lecho óseo. Debían disponer además de las medidas en milímetros de la pérdida ósea promedio experimentada por el hueso alveolar en relación al implante junto con la desviación estándar respectiva.

Los estudios de Artzi (2009), Gilliot (2011) y Martens (2014), fueron rechazados por no cumplir con los intervalos de tiempo en la realización de los controles (seguimientos). En los casos de Wolfinger et al. y Romanos (2012) se excluyeron por no utilizar los sistemas de implantación a comparar la presente revisión sistemática. El estudio de Pieri et al. (2009), no fue analizado debido a que era un estudio de serie de casos.

Las investigaciones seleccionadas se caracterizan por el análisis inicial de los criterios de inclusión, el tipo de intervención efectuada y el outcome (Tabla I.)

\section{Evaluación del éxito y fracaso:}

Outcome: Fracaso de implantes. En este análisis se incluyeron 4 estudios, con un total de 413 implantes, de los cuales 206 fueron posicionados inmediatamente después de la extracción. Se observó que en éste grupo los implantes que fracasaron ocurrieron en el grupo control (posicionamiento tardío). La presencia de heterogeneidad fue evaluada utilizando un Chi2 con un intervalo de confianza del $95 \%$. Se obtuvo un valor p de 0,03 , lo que indica heterogeneidad. EI 12 indicó que ésta es de un $65 \%$, denotando moderada heterogeneidad. La prueba de efecto global Z, permitió comprobar la presencia de homogeneidad, obteniéndose un $p$ valor de 0,34 demostrando la presencia de heterogeneidad en el estudio.
El Forest Plot (Fig. 1) mostró una clara tendencia hacia el posicionamiento tardío, lo que indica una menor pérdida de implantes al usar este protocolo. Pese a lo anterior se observó que parte del rombo quedó ubicada también en la zona de posicionamiento inmediato, así como los intervalos de confianza de Deng et al., Peñarrocha-Oltra et al. (2013) y el de Pieri et al. Esto significó a pesar que existió mejores resultados al momento de efectuar el posicionamiento de los implantes en alveolos cicatrizados, también existe la posibilidad de tener éxito al implantarlos inmediatamente después de la exodoncia.

Los cuadrados indican el peso de cada estudio, que tan importantes fueron para el análisis. Las líneas horizontales corresponden al intervalo de confianza (95\%). La medida de resumen indica la recomendación clínica

Análisis de sensibilidad. Una vez realizado el metaanálisis se procedió analizar el Funnel Plot correspondiente con el objetivo de evaluar los estudios sesgados. Se observó que el estudio de Grunder se encontraba fuera del cono, indicando su sesgo, por lo que se justifica el ajuste del metaanálisis y la utilización de la prueba de sensibilidad eliminando este artículo (Fig. 2).

La gráfica muestra cada uno de los estudios incorporados en el metaanálisis donde se analiza el sesgo de publicación. En la primera gráfica, se observa el estudio de Grunder ubicado fuera del cono, lo indica sesgo. Al ser eliminado dicho estudio del metaanálisis, se realizó nuevamente el análisis del sesgo demostrando la ausencia del mismo.

Tras realizar el análisis de sensibilidad se apreció una clara tendencia hacia la técnica de posicionamiento inmediato de implantes para obtener una menor cantidad de fracasos. No obstante en los tres estudios analizados continuó observándose que los intervalos de confianza sobrepasaban hacia la zona de posicionamiento inmediato, por lo que continuó reconociéndose la posibilidad de éxito al realizar esta técnica. En el meta análisis se evaluó la heterogeneidad utilizando la prueba de $\mathrm{Chi}^{2}$ con un intervalo de confianza de $95 \%$, éste arrojó un valor $p=0,48$, lo que denotó la ausencia de heterogeneidad, comprobada con el 12 que mostró un $0 \%$ de heterogeneidad y con la prueba de efecto global $Z$ que arrojó un valor $p=0,02$ indicando la homogeneidad presente en el análisis. (Fig. 3). 


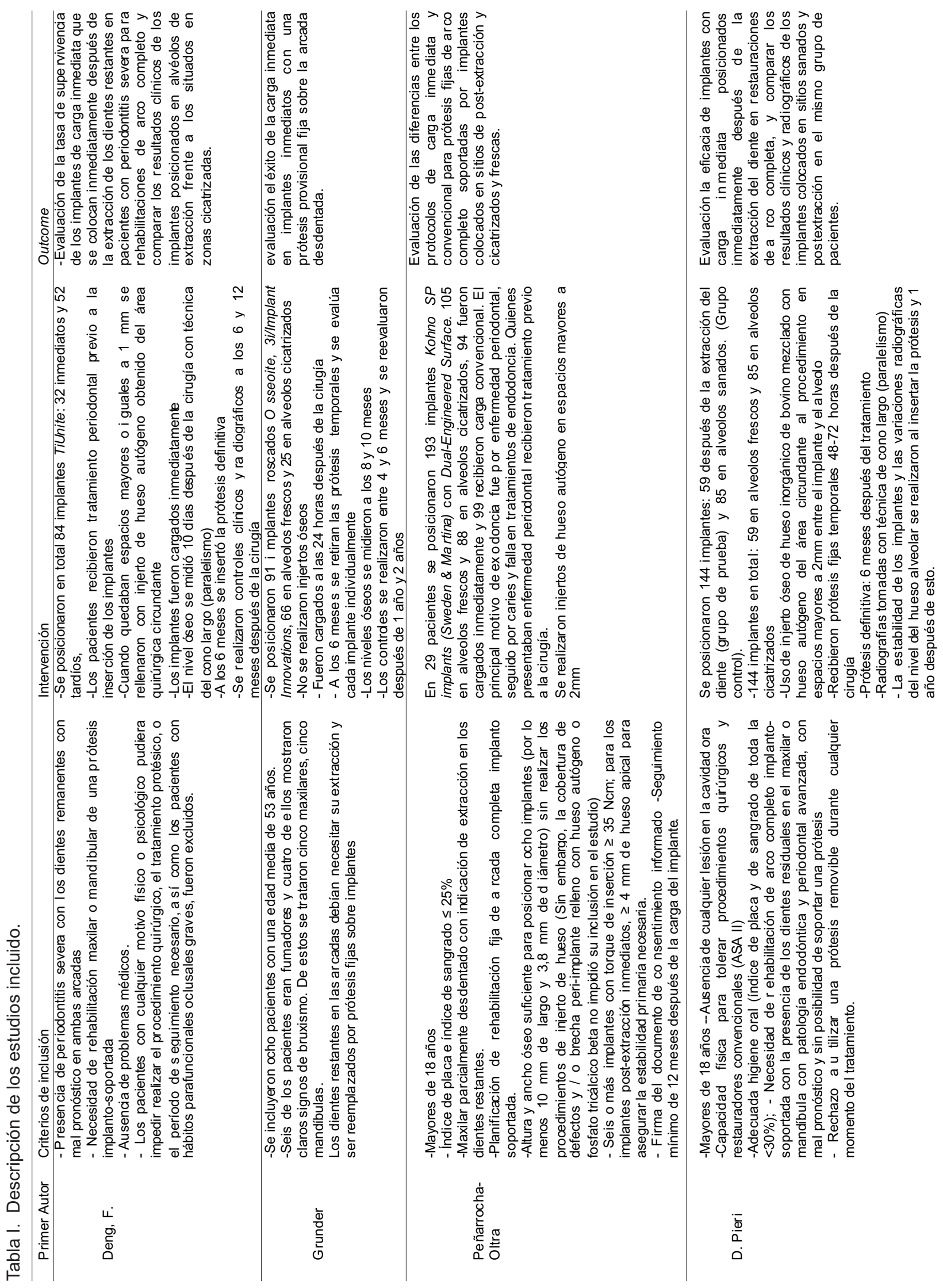


GUERRERO, M. D.; CID, G.; OLIVA, P. Evaluación de la pérdida ósea y del éxito / fracaso de implantes de titanio de posicionamiento inmediato y tardío con carga inmediata, usados para restauraciones de arco completo a 12 meses de su implantación. Int. J. Odontostomat., 10(3):399-407, 2016.

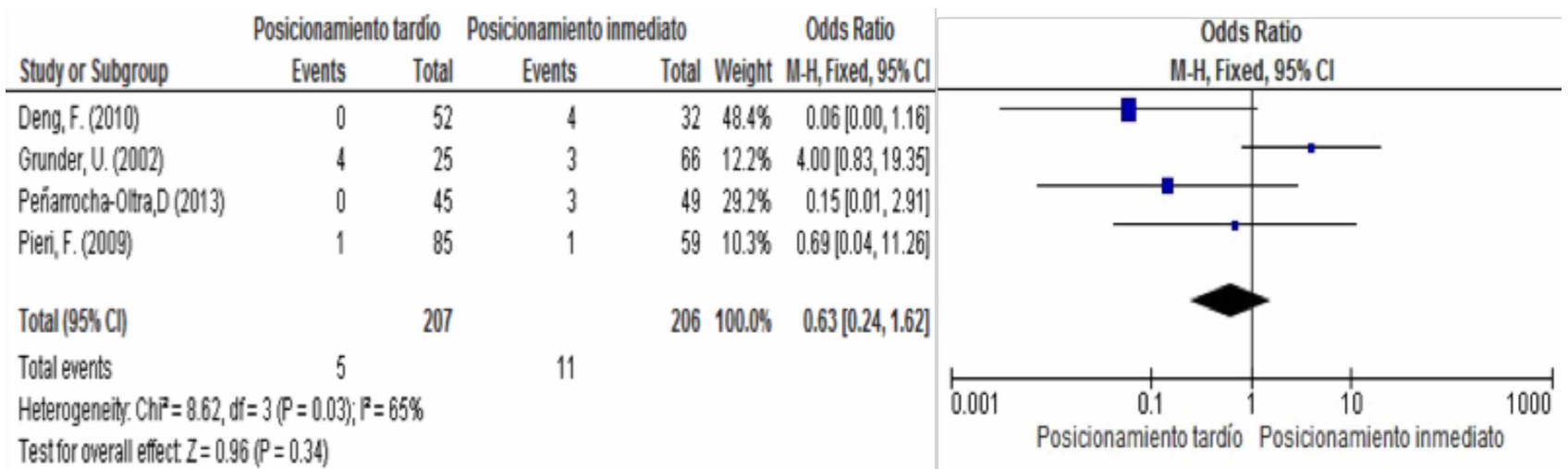

Fig. 1. Forest Plot: Evaluación de éxito y fracaso.
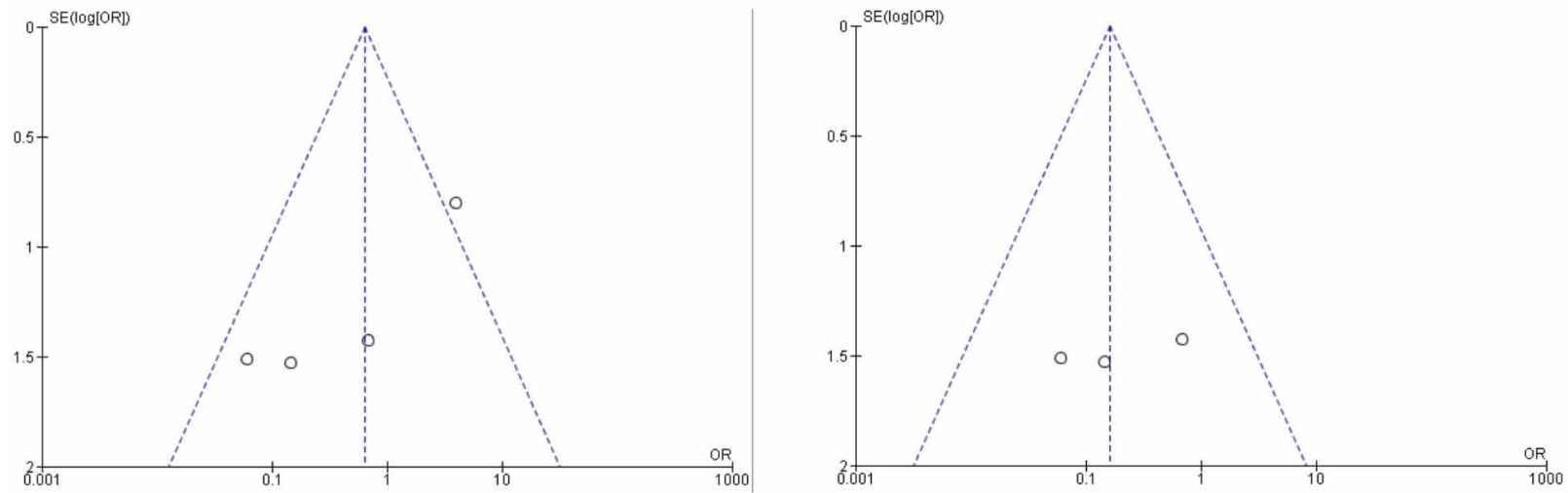

Fig. 2. Funnel Plot: análisis de sensibilidad.

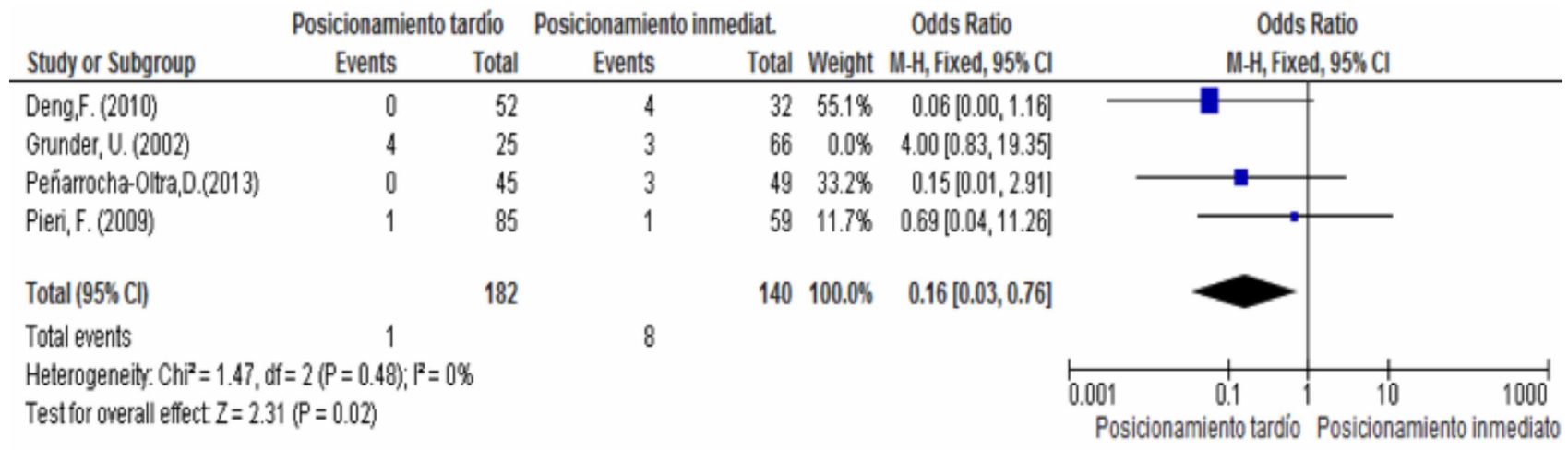

Fig. 3. Ajuste Forest Plot de evaluación éxito y fracaso tras análisis de sensibilidad.

\section{Evaluación de pérdida ósea:}

Pérdida ósea experimentada a 12 meses desde la implantación $(\mathrm{PO})(\mathrm{mm})$. Se incluyeron para este análisis los mismos estudios usados en el primer metaanálisis, pero se excluyó el estudio de Grunder, pues éste realizaba los controles del nivel óseo entre 8-10 meses tras la implantación, utilizando medidas a intervalos de $1 \mathrm{~mm}$ y entregando valores en porcentajes de cuantos implantes habían quedado en cada 
GUERRERO, M. D.; CID, G.; OLIVA, P. Evaluación de la pérdida ósea y del éxito / fracaso de implantes de titanio de posicionamiento inmediato y tardío con carga inmediata, usados para restauraciones de arco completo a 12 meses de su implantación. Int. J. Odontostomat., 10(3):399-407, 2016.

Fig. 4: Forest Plot: Evaluación de pérdida ósea.

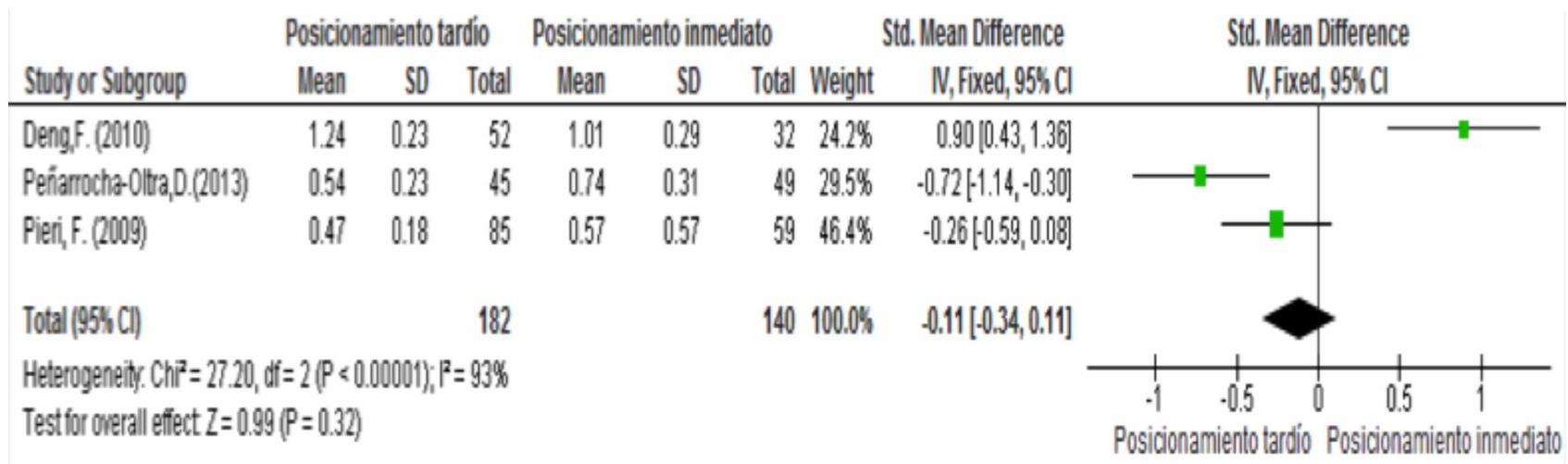

grupo establecido. Se analizaron 322 implantes, 140 de los cuales se posicionaron después de la extracción de los dientes. En este grupo se perdió un promedio de 0,11 mm en comparación con el grupo control. En el análisis se utilizó el test de Chi2 con un intervalo de confianza del $95 \%$, obteniéndose un valor $p=0,03$, lo que indica heterogeneidad. El I2 indicó que ésta equivale a un $93 \%$ lo que se traduce en alta heterogeneidad. La prueba de efecto global $Z$, permitió corroborar la ausencia de homogeneidad pues se obtuvo un $\mathrm{p}$ valor de 0,32. Pese a la alta heterogeneidad (93\%), no fue posible realizar el análisis de sensibilidad debido a la falta de más estudios que cumplieran con los criterios de inclusión para poder contrastar. (Fig. 4)

La medida de resumen indicó que la elección clínica más favorable sería el posicionamiento tardío del implante; de ésta forma las pérdidas óseas serían menores en comparación a la técnica de posicionamiento inmediato con carga funcional inmediata de implantes. Si bien los estudios de Peñarrocha-Oltra et al. y Pieri et al. avalaron esto, se observó que tanto el intervalo de confianza de éste último como la medida de resumen tenían una leve presencia hacia el posicionamiento inmediato, por lo que se concluyó que también éste protocolo podría ser clínicamente efectivo.

La pérdida ósea promedio observada en el análisis de las tres publicaciones fue de 0,75 $\mathrm{mm}$ en el grupo de posicionamiento tardío con una desviación estándar promedio de $0,21 \mathrm{~mm}$, mientras que para el grupo en que se posicionaron los implantes inmediatamente después de la exodoncia la pérdida ósea promedio tuvo un valor de $0,77 \mathrm{~mm}$ con una desviación estándar promedio de 0,39 mm a un año del primer años después de la implantación.

\section{DISCUSIÓN}

Evaluación Éxito y fracaso. Tras la evaluación de los resultados obtenidos del metaanálisis para el estudio del éxito y fracaso de los implantes respecto a su posicionamiento (inmediato y tardío), los estudios de Pieri et al., Deng et al. y Peñarrocha-Oltra et al. mostraron una mayor tendencia al fracaso en los implantes posicionados inmediatamente después de la extracción. Contrastando esto, Grunder registró 7 implantes perdidos, 4 de los cuales habían sido posicionados en alvéolos cicatrizados; demostrando una tendencia inversa a lo observado en los otros estudios.

Pieri et al. y Deng et al. a su vez concluyen que el protocolo de posicionamiento inmediato, también podría ser viable y exitoso. Deng et al. indicó que podrían obtenerse resultados protésicos satisfactorios al usar éste mismo método. Pieri et al. encontraron resultados clínicos y radiográficos similares en ambos protocolos. Coincidentemente, Peñarrocha-Oltra et al. no encontró diferencias significativas con respecto al éxito entre ambos protocolos, agregando que las complicaciones biológicas y protésicas eran extrañas para ambos grupos.

Tras realizar el análisis de sensibilidad y el correspondiente ajuste, Deng et al. y Peñarrocha-Oltra et al. coinciden con lo anteriormente analizado, con un $12,5 \%$ y un $6,1 \%$ de fracaso, respectivamente, en los implantes posicionados en alveolos frescos y un $0 \%$ de fracaso en ambos estudios en los alveolos cicatrizados. Valores similares fueron encontrados en el estudio de Pieri et al., favoreciendo también el posicionamiento tardío. 
GUERRERO, M. D.; CID, G.; OLIVA, P. Evaluación de la pérdida ósea y del éxito / fracaso de implantes de titanio de posicionamiento inmediato y tardío con carga inmediata, usados para restauraciones de arco completo a 12 meses de su implantación. Int. J. Odontostomat., 10(3):399-407, 2016.

En el presente estudio, se analizaron 413 implantes, 16 de ellos fracasaron, 11 de los cuales habían sido posicionados inmediatamente luego de la exodoncia, comprobándose una mayor tendencia al fracaso de los implantes al usar este protocolo.

Evaluación de la pérdida ósea. La pérdida ósea promedio registrada tras la realización del metaanálisis correspondió a $0,75 \pm 0,21 \mathrm{~mm}$ durante el primer año en el grupo de posicionamiento tardío y de $0,77 \pm 0,39 \mathrm{~mm}$ en el grupo de posicionamiento inmediato, pese a la similitud en los resultados, se observa una desviación estándar promedio mayor en el segundo grupo y la medida de resumen se inclina a la opción del posicionamiento tardío, lo que se corresponde a lo observado en el metaanálisis, en el cual se concluye que hubo una menor pérdida ósea al utilizar el protocolo de posicionamiento tardío, pero que también hay posibilidad de éxito al utilizar la implantación inmediata. De ésta forma, Pieri et al. y Peñarrocha-Oltra et al. mostraron una mayor tendencia a la pérdida ósea del hueso alveolar en relación a los implantes, con valores respectivos de $0,57 \pm 0,57 \mathrm{~mm}$ y $0,74 \pm 0,31 \mathrm{~mm}$, en el grupo de implantes con posicionamiento inmediato. Por el contrario, en el estudio de Deng et al. se observó una mayor pérdida de hueso en los implantes posicionados en alvéolos cicatrizados 1,24 $\pm 0,23 \mathrm{~mm}$ respecto a su contraparte, la que presentó una pér-

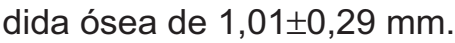

\section{CONCLUSIÓN}

Para rehabilitaciones implanto-soportadas de arco completo fijas o removibles, el protocolo de posicionamiento tardío, demostró ser una mejor alternativa puesto que manifestó una menor tasa de fracaso y una menor pérdida ósea. No obstante, no se rechaza la viabilidad del posicionamiento inmediato. Aún faltan estudios para poder guiar con exactitud las decisiones de los clínicos, al momento de decidir el protocolo seguir.

GUERRERO, M. D.; CID, G. \& OLIVA, P.Evaluation of bone loss and success / failure of titanium implant positioning with immediate and late charge, used in full arch restorations at 12 months of implant. Int. J. Odontostomat., 10(3):399-407, 2016.

ABSTRACT: The purpose of this study was to evaluate the bone level and the success and failure of loaded implants positioned immediately after extraction for full-arch restorations and compare the results obtained with implants placed in healed alveoli, in a period of 12 months, to determine the best alternative. Asystematic review based on prospective randomized clinical trials, and randomized or nonrandomized controlled and uncontrolled trials evaluating failure and bone resorption of immediate loaded implants, positioned in post extraction and healed sockets in adult patients for full arch rehabilitations. Data from clinical trials were entered into Review Manager® software. Two meta-analyzes for different variables to analyze and subsequently a sensitivity analysis to eliminate bias caused studies were performed. From a total of 431 implants studied, 16 of them failed, 11 of which had been positioned immediately after the extraction. Three of the authors of the review considered agree that there are more successful and less bone loss in implants positioned so late. To implantsupported full-arch restorations fixed or removable, late positioning protocol proved to be a better alternative since it showed a lower failure rate and less bone loss.

KEY WORDS: restoring full arch, dental implant, alveolar resorption, immediate loading, immediate position, late position.

\section{REFERENCIAS BIBLIOGRÁFICAS}

Barbier, L.; Abeloos, J.; De Clercq, C. \& Jacobs, R. Peri-implant bone changes following tooth extraction, immediate placement and loading of implants in the edentulous maxilla. Clin. Oral Invest., 16(4):1061-79, 2012.

Deng, F.; Zhang, H.; Zhang, H.; Shao, H.; He, Q. \& Zhang, P. A comparison of clinical outcomes for implants placed in fresh extraction sockets versus healed sites in periodontally compromised patients: a 1-year follow-up report. Int. J. Oral Maxillofac. Implants, 25(5):1036-40, 2010.

Esposito, M.; Grusovin, M. G.; Polyzos, I. P.; Felice, P. \& Worthington, H. V. Interventions for replacing missing teeth: dental implants in fresh extraction 
GUERRERO, M. D.; CID, G.; OLIVA, P. Evaluación de la pérdida ósea y del éxito / fracaso de implantes de titanio de posicionamiento inmediato y tardío con carga inmediata, usados para restauraciones de arco completo a 12 meses de su implantación. Int. J. Odontostomat., 10(3):399-407, 2016.

sockets (immediate, immediate-delayed and delayed implants). Cochrane Database Syst. Rev., (9):CD005968, 2010.

Esposito, M.; Grusovin, M. G.; Willings, M.; Coulthard, P. \& Worthington, H. V. The effectiveness of immediate, early, and conventional loading of dental implants: a Cochrane systematic review of randomized controlled clinical trials. Int. J. Oral Maxillofac. Implants, 22(6):893-904, 2007.

Grunder, U. Immediate functional loading of immediate implants in edentulous arches: two-year results. Int. J. Periodontics Restorative Dent., 21(6):545-51, 2001.

Jiménez-López, V. Rehabilitación Oral en Prótesis sobre Implante. Su Relación con la Estética, Oclusión, A. T. M., Ortodoncia, Fonética y Laboratorio. Barcelona, Quintessence, 1998.

Peñarrocha, M.; Uribe, R. \& Balaguer, J. Implantes inmediatos a la exodoncia. Situación actual. Med. Oral Patol. Oral Cir. Bucal, 9(3):234-42, 2004.

Peñarrocha-Oltra, D.; Covani, U.; Aparicio, A.; Ata-Ali, J.; Peñarrocha-Diago, M. \& Peñarrocha-Diago, M. Immediate versus conventional loading for the maxilla with implants placed into fresh and healed extraction sites to support a full-arch fixed prosthesis: nonrandomized controlled clinical study. Int. J. Oral Maxillofac. Implants, 28(4):1116-24, 2013.

Pieri, F.; Aldini, N. N.; Fini, M. \& Corinaldesi, G. Immediate occlusal loading of immediately placed implants supporting fixed restorations in completely edentulous arches: a 1-year prospective pilot study. J. Periodontol., 80(3):411-21, 2009.

Sáenz, C.; Torres, D. \& Gutiérrez, J. Osteointegración e implantes bucales. Secib Online, 3(1):22-7, 2006.

Spiekermann, H.; Donath, K.; Jovanovic, S. \& Richter, J. Atlas de Implantología. Barcelona, Masson, 2001.

Venegas-Acosta, J. C.; Landínez-Parra, N. S. \& GarzónAlvarado, D. A. Mecanobiología de la interfase huesoimplante dental. Rev. Cuba. Estomatol., 47(1):14-36, 2010.

Wolfinger, G. J.; Balshi, T. J. \& Rangert, B. Immediate functional loading of Brånemark system implants in edentulous mandibles: clinical report of the results of developmental and simplified protocols. Int. J. Oral Maxillofac. Implants, 18(2):250-7, 2003.
Correspondence to:

Dr. Patricio Oliva

Facultad de Ciencias de la Salud

Universidad del Desarrollo

Concepción

CHILE

Email: patricioolivamella@gmail.com

Received: 18-01-2015

Accepted: 06-10-2016 